\title{
The PUA representations of the Shubnikov point groups
}

\author{
P M van den Broek \\ Institute for Theoretical Physics, University of Nijmegen, Nijmegen, The Netherlands
}

Received 14 August 1978

Abstract. The irreducible PUA representations of the Shubnikov point groups are tabulated using the procedure of generalised induction and the decomposition theory of factor systems.

\section{Introduction}

It is by now well known that in the quantum mechanical description of a physical system an essential role is played by the projective unitary-antiunitary (PUA) representations of of the symmetry group of the system. The PUA representations of the Shubnikov space groups are important if one considers for instance a charged particle in a crystalline potential with an external magnetic field (Tam 1969).

The irreducible PUA representations of the Shubnikov space groups may be derived by the procedure of generalised induction (Shaw and Lever 1974, van den Broek 1977) starting from the subgroup of translations. To apply this procedure one has to know the irreducible PUA representations of the Shubnikov point groups.

The irreducible UA representations of the Shubnikov point groups have been given by Bradley and Cracknell (1972). Hurley (1966) has tabulated the irreducible PU representations of the ordinary point groups. A complete tabulation of the irreducible PUA representations of the Shubnikov point groups has however not previously been given. To provide this tabulation will be the aim of this paper.

Since the number of inequivalent factor systems of the Shubnikov point groups may be very large (up to 128) it would take a lot of space to give for each Shubnikov point group the irreducible PUA representations for each of the inequivalent factor systems. Instead we will only give in our tabulation the material which will enable the reader to construct the factor systems and the irreducible PUA representation himself, using the procedure of generalised induction and the decomposition theory of factor systems (van den Broek 1976).

We will first treat those Shubnikov point groups which are semidirect products where the invariant subgroup is a cyclic subgroup of the non-magnetic subgroup. The groups which fulfil this requirement are said to be of type A. Starting from a cyclic non-magnetic subgroup has the advantage that it has only trivial factor systems and one-dimensional irreducible unitary representations. The requirement that the Shubnikov point group is a semidirect product leads to an enormous simplification of the labelling of the inequivalent factor systems.

There are 47 Shubnikov point groups, if isomorphic groups whose non-magnetic subgroups are also isomorphic are identified. The five cyclic ordinary point groups need 
of course not to be treated. Of the other 42 Shubnikov point group (listed in table 1) 34 are of type A. The procedure of generalised induction and the decomposition theory of factor systems for these groups is described briefly in $\S 3$. Tables for the irreducible PUA representations of these groups are given in $\$ 4$.

Among the remaining eight Shubnikov point groups there are two ordinary point groups: $\mathrm{T}$ and $\mathrm{O}$. Although their irreducible $\mathrm{PU}$ representations are already known (Hurley 1966, Bradley and Cracknell 1972) they are given in $\S 5$ for the sake of completeness. For the remaining six Shubnikov point groups, which are said to be of type B, we may assume now that the irreducible PU representations of their non-magnetic subgroups are known. In $\S 6$ we will describe briefly how the irreducible PUA representations of a Shubnikov point group whose non-magnetic subgroup is of index 2 can be obtained from the irreducible PU representations of the non-magnetic subgroup. Tables for the irreducible PUA representations of the Shubnikov point groups of type B are given in $\S 7$. Finally we discuss in the Appendix the problem of how to find a relation between an arbitrary factor system and the factor systems used in the tabulation. But first we give in the next section some mathematical preliminaries.

\section{Preliminaries}

Let $G$ be a finite group and $G_{0}$ a subgroup of $G$ of index 1 or 2, called the non-magnetic subgroup of $G$. A PUA representation of $G$ with respect to $G_{0}$ is a mapping $D$ from $G$ to the complex unitary matrices of some dimension $n$ such that:

(i)

$$
D(e)=I
$$

where $e$ is the identity of $G$ and $I$ is the $n$-dimensional unit matrix;

$$
D(g) D^{g}\left(g^{\prime}\right)=\sigma\left(g, g^{\prime}\right) D\left(g g^{\prime}\right) \quad \forall g, g^{\prime} \in G
$$

for some mapping $\sigma: G \times G \rightarrow U(1)$. Here $D^{g}$ is defined by

$$
D^{g}= \begin{cases}D & \text { if } g \in G_{0} \\ D^{*} & \text { if } g \notin G_{0}\end{cases}
$$

and the asterisk denotes complex conjugation. The mapping $\sigma$ is called a factor system of $G$ with respect to $G_{0}$ and satisfies

$$
\sigma(g, e)=\sigma(e, g)=1 \quad \forall g \in G
$$

and

$$
\sigma\left(g, g^{\prime}\right) \sigma\left(g g^{\prime}, g^{\prime \prime}\right)=\sigma\left(g, g^{\prime} g^{\prime \prime}\right) \sigma^{g}\left(g^{\prime}, g^{\prime \prime}\right) \quad \forall g, g^{\prime}, g^{\prime \prime} \in G
$$

where $\sigma^{g}$ is defined as in equation (2.3). In the following we will drop the phrase "with respect to ...' wherever it is clear which is the appropriate non-magnetic subgroup.

A factor system $\sigma$ of $G$ is called trivial if it can be written as

$$
\sigma\left(g, g^{\prime}\right)=c(g) c^{g}\left(g^{\prime}\right) c^{-1}\left(g g^{\prime}\right) \quad \forall g, g^{\prime} \in G
$$

for some mapping $c: G \rightarrow U(1)$. Two factor systems $\sigma$ and $\sigma^{\prime}$ are called equivalent if $\sigma^{\prime} \sigma^{-1}$ is trivial. Two PUA representations $D_{1}$ and $D_{2}$ are equivalent if there exists a unitary matrix $S$ such that

$$
D_{1}(g)=S^{-1} D_{2}(g) S^{g} \quad \forall g \in G
$$


A PUA representation $D$ of $G$ is reducible if $D$ is equivalent with a PUA representation $D^{\prime}$ which can be written in the form

$$
D^{\prime}(g)=\left(\begin{array}{ll}
D_{1}(g) & 0 \\
0 & D_{2}(g)
\end{array}\right) \quad \forall g \in G
$$

otherwise $D$ is irreducible.

\section{Generalised induction and decomposition of factor systems for Shubnikov point groups of type $\mathbf{A}$}

Suppose that $G$ is a semidirect product:

$$
G=H \wedge K
$$

where $H$ is a cyclic subgroup of $G_{0}$. Elements of $G$ are denoted by $h k$ where $h \in H$ and $k \in K$. Since $H$ has only trivial factor systems, each factor system of $G$ is equivalent with a factor system of the form

$$
\sigma\left(h k, h^{\prime} k^{\prime}\right)=v\left(k, k^{\prime}\right) P_{k}\left(h^{\prime}\right)
$$

where $v$ is a factor system of $K$ with respect to $K_{0}=G_{0} / H$ and $\left\{P_{k}\right\}$ is a set of one-dimensional representations of $H$ which satisfy

$$
P_{k k^{\prime}}(h)=P_{k^{\prime}}\left(k^{\prime} h k^{\prime-1}\right) P_{k^{\prime}}^{k}(h) \quad \forall h \in H, \forall k, k^{\prime} \in K .
$$

(This follows from theorem 1 of van den Broek (1976)).

If $\sigma$ and $\sigma^{\prime}$ are two factor systems of $G$ with the above property: $\sigma\left(h k, h^{\prime} k^{\prime}\right)=$ $v\left(k, k^{\prime}\right) P_{k}\left(h^{\prime}\right)$ and $\sigma^{\prime}\left(h k, h^{\prime} k^{\prime}\right)=v^{\prime}\left(k, k^{\prime}\right) P_{k}^{\prime}\left(h^{\prime}\right)$ then $\sigma$ and $\sigma^{\prime}$ are equivalent if and only if $v$ and $v^{\prime}$ are equivalent and there exists a one-dimensional representation $Q$ of $H$ such that

$$
P_{k}^{\prime}(h) P_{k}^{*}(h)=Q^{k}(h) Q^{*}\left(k h k^{-1}\right) \quad \forall h \in H, \forall k \in K
$$

(This follows from theorem 3 of van den Broek (1976)).

Therefore we define two solutions $\left\{P_{k}\right\}$ and $\left\{P_{k}^{\prime}\right\}$ of equation (3.3) to be equivalent if a $Q$ exists such that equation (3.4) holds. A complete set of of inequivalent factor systems of $G$ is then given by equation (3.2) if $v$ runs through a complete set of inequivalent factor systems of $K$ and $P$ runs through a complete set of inequivalent solutions of equation (3.3).

The procedure of generalised induction now goes as follows (Shaw and Lever 1974, van den Broek 1977). Consider a factor system $\sigma$ of $G$ which satisfies equation (3.2). For each irreducible representation $\Gamma$ of $H$, which is one-dimensional since $H$ is abelian, the representation $\Gamma_{k}$ of $H$ is defined for each $k \in K$ by

$$
\Gamma_{k}(h)=\Gamma^{k}\left(k^{-1} h k\right) P_{k}^{*}\left(k^{-1} h k\right) \quad \forall h \in H
$$

and the orbit of $\Gamma$ is defined to be $\left\{\Gamma_{k} \mid k \in K\right\} . M(\Gamma)$ is the subgroup of $K$ defined by

$$
M(\Gamma)=\left\{k \in K \mid \Gamma_{k}=\Gamma\right\}
$$

and the little group $L(\Gamma)$ is defined by

$$
L(\Gamma)=\{h k \mid h \in H, k \in M(\Gamma)\} .
$$




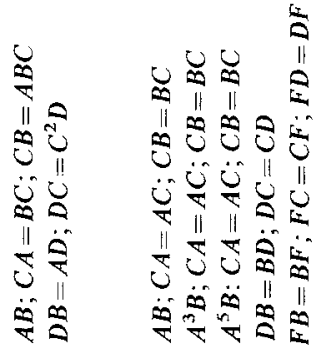

₹ै

II II II

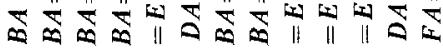

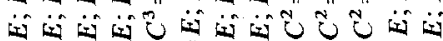

|| || || || || || || || || || || ||

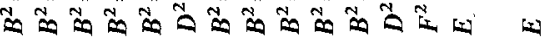

il II II II

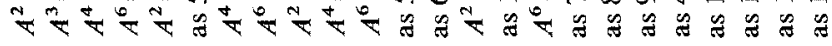

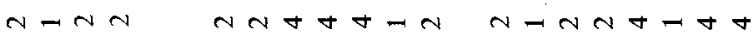

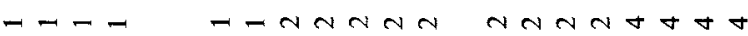

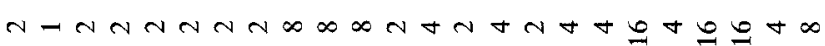

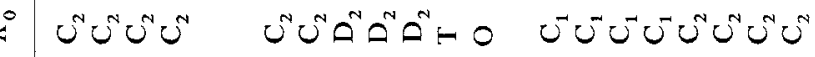

$\frac{\measuredangle}{5}$

$\cup$ ن

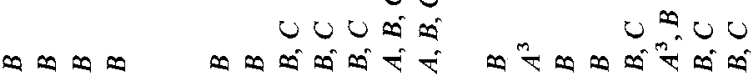



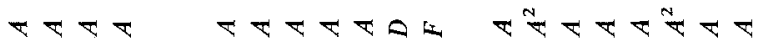

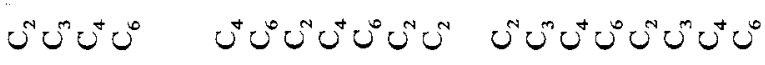

$\approx$

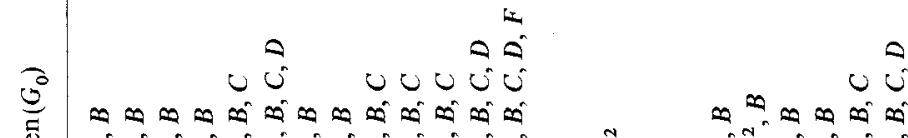

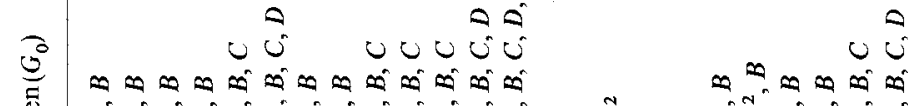

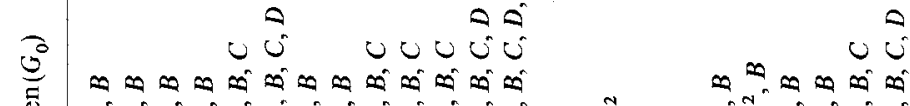

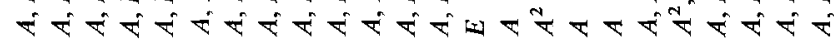
$U_{x}^{N} U_{x}^{N} U_{x}^{N} U_{x}^{N} U_{x}^{N} U_{x}^{N} U_{x}^{N}$

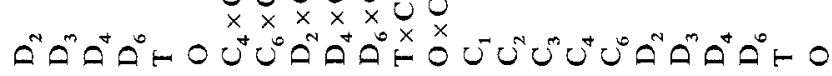

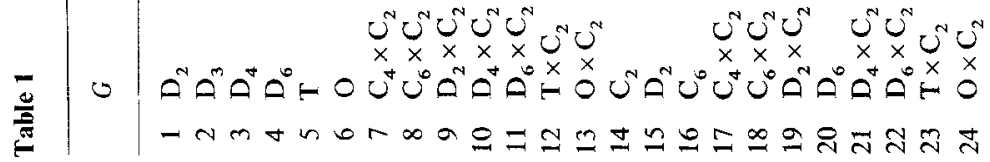




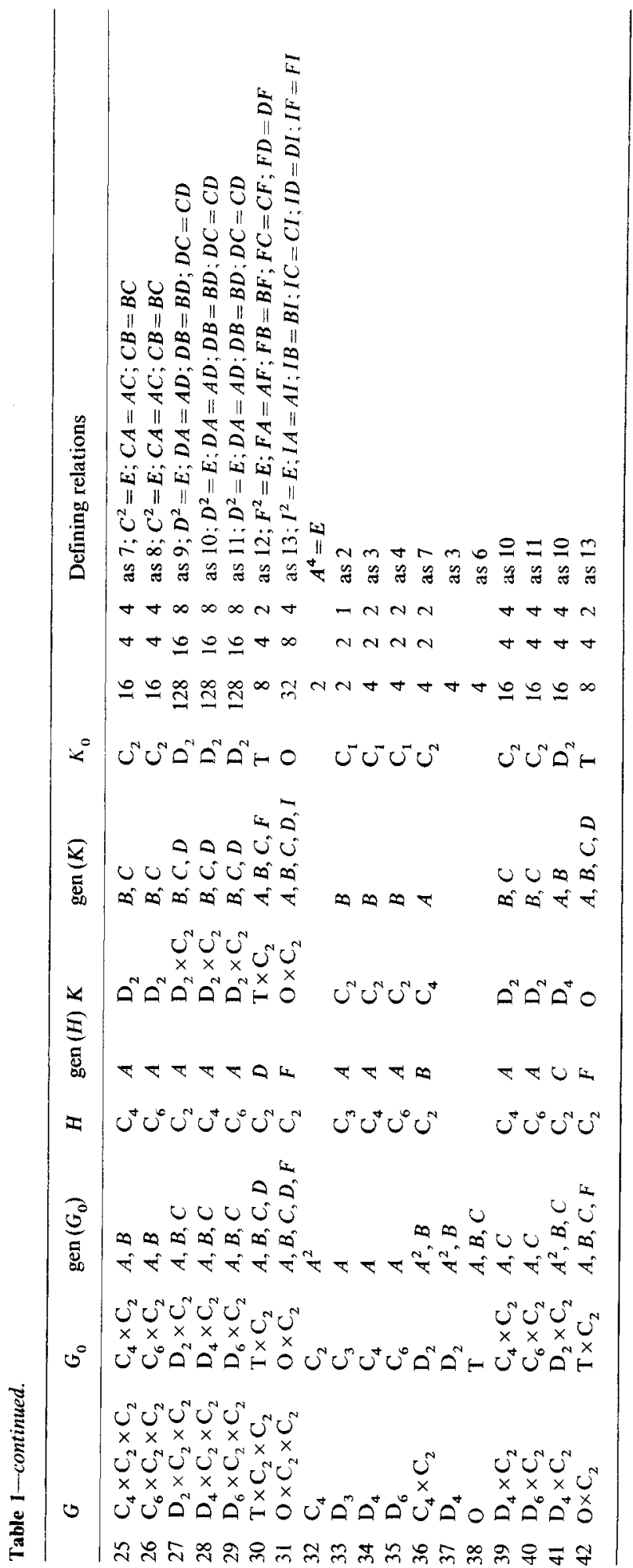


The allowable PUA representations of $L(\Gamma)$ are defined by

$$
D(h k)=\Gamma(h) \Delta(k)
$$

where $\Delta$ runs through the irreducible PUA representations of $M(\Gamma)$ with factor system $v$. Suppose

$$
G=\sum_{i=1}^{p} g_{i} L(\Gamma)
$$

where $\left\{g_{1}=e, g_{2}, \ldots g_{p}\right\}$ is a set of left coset representatives of $G$ with respect to $L(\Gamma)$. If $D$ is a PUA representation of $L(\Gamma)$ with factor system $\sigma$ then the induced PUA representation $D \uparrow G$ of $G$ is defined by

$$
(D \uparrow G)_{j t, k s}(g)= \begin{cases}0 & \text { if } g_{j}^{-1} g g_{k} \notin L(\Gamma) \\ \sigma\left(g, g_{k}\right) \sigma^{*}\left(g_{j}, g_{j}^{-1} g g_{k}\right) D_{t s}^{g_{j}}\left(g_{j}^{-1} g g_{k}\right) & \text { if } g_{j}^{-1} g g_{k} \in L(\Gamma)\end{cases}
$$

and belongs also to the factor system $\sigma$. A complete set of irreducible PUA representations of $G$ with factor system $\sigma$ is obtained by inducing all the allowable PUA representations of $L(\Gamma)$ to $G$ for one representative $\Gamma$ of each orbit

Now it is clear what information is needed in order to be able to write down the matrices of all the irreducible PUA representations of $G$ :

(i) a complete set of inequivalent solutions of equation (3.3);

(ii) for each such solution a set of representatives of the orbits of the irreducible unitary representations of $H$;

(iii) for each such representative $\Gamma$ the group $M(\Gamma)$;

(iv) for each group $M(\Gamma)$ all its irreducible PUA representations.

For each irreducible PUA representation $\Delta$ of $M(\Gamma)$ one obtains an irreducible PUA representation $D \uparrow G$ with the equations (3.8) and (3.10). The factor system $\sigma$ of $D \uparrow G$ is given by equation (3.2) where $v$ is the factor system of $\Delta$.

The information (i), (ii) and (iii) will be tabulated in the next section for each Shubnikov point group of type A. It is supposed that the irreducible PUA representations of the groups $M(\Gamma)$ are already known. In some cases however they must be determined with the methods described in this paper; so then one has to apply these methods more than once.

\section{Tables for the irreducible PUA representations of the Shubnikov point groups of type A}

In table 1 all Shubnikov point groups $G$ with their non-magnetic subgroups $G_{0}$ and their defining relations are given. $E$ always denotes the identity of $G$. Of the Shubnikov point groups of type A also the cyclic invariant subgroup $H, K=G / H$, and $K_{0}=G_{0} / H$ are given. Also the generators of $G_{0}, H$ and $K$ are given. In the three columns after the column of $K_{0}$ we give respectively the number of inequivalent factor systems of $G$, the number of inequivalent factor systems of $K$ and their quotient, which is the number of inequivalent solutions of equation (3.3).

The tables 2.1-2.42, where the last number corresponds to the number in the first column of table 1 of the group under consideration, contain the following information: the inequivalent solutions of equation (3.3) are given in the first column, where for each generator of $K$ an irreducible representation of $H$ is given; for each of the inequivalent solutions of equation (3.3) the second column gives representatives of each orbit of irreducible representations of $H$ together with the generators of the corresponding groups $M(\Gamma)$. 
Table 2.1

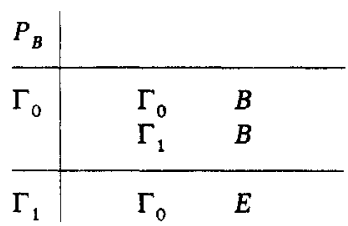

Table 2.2

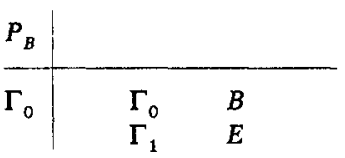

Table 2.4

\begin{tabular}{l|ll}
$P_{B}$ & & \\
\hline$\Gamma_{0}$ & $\Gamma_{0}$ & $B$ \\
& $\Gamma_{1}$ & $E$ \\
& $\Gamma_{2}$ & $E$ \\
& $\Gamma_{3}$ & $B$ \\
\hline$\Gamma_{3}$ & $\Gamma_{0}$ & $E$ \\
& $\Gamma_{2}$ & $E$ \\
& $\Gamma_{4}$ & $E$
\end{tabular}

Table. 2.7

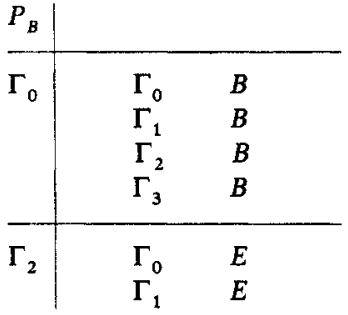

Table 2.3

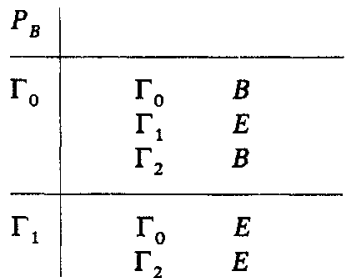

Table 2.8

\begin{tabular}{c|cc}
$P_{B}$ & & \\
\hline$\Gamma_{0}$ & $\Gamma_{0}$ & $B$ \\
& $\Gamma_{1}$ & $B$ \\
& $\Gamma_{2}$ & $B$ \\
& $\Gamma_{3}$ & $B$ \\
& $\Gamma_{4}$ & $B$ \\
& $\Gamma_{5}$ & $B$ \\
\hline$\Gamma_{1}$ & $\Gamma_{0}$ & $E$ \\
& $\Gamma_{1}$ & $E$ \\
& $\Gamma_{2}$ & $E$
\end{tabular}

Table 2.9

\begin{tabular}{ll|ll}
$P_{B}$ & $P_{C}$ & & \\
\hline$\Gamma_{0}$ & $\Gamma_{0}$ & $\Gamma_{0}$ & $B, C$ \\
& & $\Gamma_{1}$ & $B, C$ \\
\hline$\Gamma_{0}$ & $\Gamma_{1}$ & $\Gamma_{0}$ & $B$ \\
\hline$\Gamma_{1}$ & $\Gamma_{0}$ & $\Gamma_{0}$ & $C$ \\
\hline$\Gamma_{1}$ & $\Gamma_{1}$ & $\Gamma_{0}$ & $B C$
\end{tabular}

Table 2.11

\begin{tabular}{ll|ll}
$P_{B}$ & $P_{C}$ & & \\
\hline$\Gamma_{0}$ & $\Gamma_{0}$ & $\Gamma_{0}$ & $B, C$ \\
& & $\Gamma_{1}$ & $C$ \\
& & $\Gamma_{2}$ & $C$ \\
& & $\Gamma_{3}$ & $B, C$ \\
\hline$\Gamma_{3}$ & $\Gamma_{0}$ & $\Gamma_{0}$ & $C$ \\
& & $\Gamma_{2}$ & $C$ \\
& & $\Gamma_{4}$ & $C$ \\
\hline$\Gamma_{0}$ & $\Gamma_{3}$ & $\Gamma_{0}$ & $B$ \\
& & $\Gamma_{1}$ & $E$ \\
\hline$\Gamma_{3}$ & $\Gamma_{3}$ & $\Gamma_{0}$ & $B C$ \\
& & $\Gamma_{1}$ & $E$
\end{tabular}

Table 2.10

\begin{tabular}{ll|ll}
$P_{B}$ & $P_{C}$ & & \\
\hline$\Gamma_{0}$ & $\Gamma_{0}$ & $\Gamma_{0}$ & $B, C$ \\
& & $\Gamma_{1}$ & $C$ \\
& & $\Gamma_{2}$ & $B, C$ \\
\hline$\Gamma_{1}$ & $\Gamma_{0}$ & $\Gamma_{0}$ & $C$ \\
& & $\Gamma_{2}$ & $C$ \\
\hline$\Gamma_{0}$ & $\Gamma_{2}$ & $\Gamma_{0}$ & $B$ \\
& & $\Gamma_{1}$ & $B C$ \\
\hline$\Gamma_{1}$ & $\Gamma_{2}$ & $\Gamma_{0}$ & $E$
\end{tabular}

Table 2,12

\begin{tabular}{|c|c|c|c|c|}
\hline$P_{A}$ & $P_{B}$ & $P_{C}$ & & \\
\hline$\Gamma_{\mathbf{0}}$ & $\Gamma_{0}$ & $\Gamma_{o}$ & $\begin{array}{l}\Gamma_{0} \\
\Gamma_{1}\end{array}$ & $\begin{array}{l}A, B, C \\
A, B, C\end{array}$ \\
\hline
\end{tabular}


Table 2.13

\begin{tabular}{lccc|cl}
$P_{A}$ & $P_{B}$ & $P_{C}$ & $P_{D}$ & & \\
\hline$\Gamma_{0}$ & $\Gamma_{0}$ & $\Gamma_{0}$ & $\Gamma_{0}$ & $\Gamma_{0}$ & $A, B, C, D$ \\
& & & & $\Gamma_{1}$ & $A, B, C, D$ \\
\hline$\Gamma_{0}$ & $\Gamma_{0}$ & $\Gamma_{0}$ & $\Gamma_{1}$ & $\Gamma_{0}$ & $A, B, C$
\end{tabular}

Table 2.15

\begin{tabular}{l|ll}
$P_{B}$ & & \\
\hline$\Gamma_{0}$ & $\Gamma_{0}$ & $B$ \\
& $\Gamma_{1}$ & $B$ \\
\hline$\Gamma_{1}$ & $\Gamma_{0}$ & $E$
\end{tabular}

Table 2.16

\begin{tabular}{l|ll}
$P_{A^{3}}$ & & \\
\hline$\Gamma_{0}$ & $\Gamma_{0}$ & $A^{3}$ \\
& $\Gamma_{1}$ & $E$
\end{tabular}

Table 2.17

\begin{tabular}{c|cc}
$P_{B}$ & & \\
\hline$\Gamma_{0}$ & $\Gamma_{0}$ & $B$ \\
& $\Gamma_{1}$ & $B$ \\
& $\Gamma_{2}$ & $B$ \\
\hline$\Gamma_{1}$ & $\Gamma_{0}$ & $E$ \\
& $\Gamma_{2}$ & $E$
\end{tabular}

Table 2.18

\begin{tabular}{l|ll}
$P_{B}$ & & \\
\hline$\Gamma_{0}$ & $\Gamma_{0}$ & $B$ \\
& $\Gamma_{1}$ & $E$ \\
& $\Gamma_{2}$ & $E$ \\
& $\Gamma_{3}$ & $B$ \\
\hline$\Gamma_{3}$ & $\Gamma_{0}$ & $E$ \\
& $\Gamma_{2}$ & $E$ \\
& $\Gamma_{4}$ & $E$
\end{tabular}

Table 2.19

\begin{tabular}{ll|ll}
$P_{B}$ & $P_{C}$ & & \\
\hline$\Gamma_{0}$ & $\Gamma_{0}$ & $\begin{array}{l}\Gamma_{0} \\
\Gamma_{1}\end{array}$ & $B, C$ \\
\hline$\Gamma_{0}$ & $\Gamma_{1}$ & $\Gamma_{0}$ & $B$ \\
\hline$\Gamma_{0}$ & $\Gamma_{0}$ & $\Gamma_{0}$ & $C$ \\
\hline$\Gamma_{1}$ & $\Gamma_{1}$ & $\Gamma_{0}$ & $B C$
\end{tabular}

Table 2.20

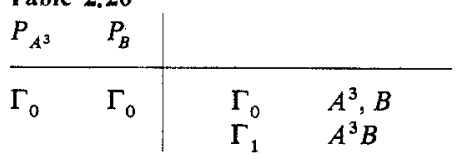

Table 2.21

\begin{tabular}{ll|ll}
$P_{B}$ & $P_{C}$ & & \\
\hline$\Gamma_{0}$ & $\Gamma_{0}$ & $\Gamma_{0}$ & $B, C$ \\
& & $\Gamma_{1}$ & $B C$ \\
& & $\Gamma_{2}$ & $B, C$ \\
\hline$\Gamma_{2}$ & $\Gamma_{0}$ & $\Gamma_{n}$ & $C$ \\
& & $\Gamma_{1}$ & $B$ \\
\hline$\Gamma_{1}$ & $\Gamma_{1}$ & $\Gamma_{0}$ & $E$ \\
\hline$\Gamma_{3}$ & $\Gamma_{1}$ & $\Gamma_{0}$ & $B C$ \\
& & $\Gamma_{1}$ & $B C$
\end{tabular}

Table 2.22

\begin{tabular}{ll|ll}
$P_{B}$ & $P_{C}$ & & \\
\hline$\Gamma_{0}$ & $\Gamma_{0}$ & $\Gamma_{0}$ & $B, C$ \\
& & $\Gamma_{1}$ & $B C$ \\
& & $\Gamma_{2}$ & $B C$ \\
& & $\Gamma_{3}$ & $B, C$ \\
\hline$\Gamma_{0}$ & $\Gamma_{3}$ & $\Gamma_{0}$ & $B$ \\
& & $\Gamma_{2}$ & $E$ \\
\hline$\Gamma_{3}$ & $\Gamma_{0}$ & $\Gamma_{0}$ & $C$ \\
& & $\Gamma_{2}$ & $E$ \\
\hline$\Gamma_{3}$ & $\Gamma_{3}$ & $\Gamma_{0}$ & $B C$ \\
& & $\Gamma_{2}$ & $B C$ \\
& & $\Gamma_{4}$ & $B C$
\end{tabular}


Table 2.25

\begin{tabular}{ll|ll}
$P_{B}$ & $P_{C}$ & & \\
\hline$\Gamma_{0}$ & $\Gamma_{0}$ & $\Gamma_{0}$ & $B, C$ \\
& & $\Gamma_{1}$ & $B$ \\
& & $\Gamma_{2}$ & $B, C$ \\
\hline$\Gamma_{0}$ & $\Gamma_{1}$ & $\Gamma_{0}$ & $B$ \\
& & $\Gamma_{1}$ & $B$ \\
\hline$\Gamma_{2}$ & $\Gamma_{0}$ & $\Gamma_{0}$ & $C$ \\
\hline$\Gamma_{2}$ & $\Gamma_{1}$ & $\Gamma_{1}$ & $B C$ \\
\hline
\end{tabular}

Table 2.26

\begin{tabular}{ll|ll}
$P_{B}$ & $P_{C}$ & & \\
\hline$\Gamma_{0}$ & $\Gamma_{0}$ & $\Gamma_{0}$ & $B, C$ \\
& & $\Gamma_{1}$ & $B$ \\
& & $\Gamma_{2}$ & $B$ \\
& & $\Gamma_{3}$ & $B, C$ \\
\hline$\Gamma_{0}$ & $\Gamma_{3}$ & $\Gamma_{0}$ & $B$ \\
& & $\Gamma_{2}$ & $B$ \\
& & $\Gamma_{4}$ & $B$ \\
\hline$\Gamma_{3}$ & $\Gamma_{0}$ & $\Gamma_{0}$ & $C$ \\
& & $\Gamma_{2}$ & $E$ \\
\hline$\Gamma_{3}$ & $\Gamma_{3}$ & $\Gamma_{0}$ & $B C$ \\
& & $\Gamma_{2}$ & $E$
\end{tabular}

Table 2.27

\begin{tabular}{lll|ll}
$P_{B}$ & $P_{C}$ & $P_{D}$ & & \\
\hline$\Gamma_{0}$ & $\Gamma_{0}$ & $\Gamma_{0}$ & $\Gamma_{0}$ & $\begin{array}{l}B, C, D \\
\Gamma_{1}\end{array}$ \\
\hline$\Gamma_{0}$ & $\Gamma_{0}$ & $\Gamma_{1}$ & $\Gamma_{0}$ & $B, C$ \\
\hline$\Gamma_{0}$ & $\Gamma_{1}$ & $\Gamma_{0}$ & $\Gamma_{0}$ & $B, D$ \\
\hline$\Gamma_{1}$ & $\Gamma_{0}$ & $\Gamma_{0}$ & $\Gamma_{0}$ & $C, D$ \\
\hline$\Gamma_{0}$ & $\Gamma_{1}$ & $\Gamma_{1}$ & $\Gamma_{0}$ & $B, C D$ \\
\hline$\Gamma_{1}$ & $\Gamma_{0}$ & $\Gamma_{1}$ & $\Gamma_{0}$ & $C, B D$ \\
\hline$\Gamma_{1}$ & $\Gamma_{1}$ & $\Gamma_{0}$ & $\Gamma_{0}$ & $D, B C$ \\
\hline$\Gamma_{1}$ & $\Gamma_{1}$ & $\Gamma_{1}$ & $\Gamma_{0}$ & $B C, C D$
\end{tabular}

Table 2,28

\begin{tabular}{lll|ll}
$P_{B}$ & $P_{C}$ & $P_{D}$ & & \\
\hline$\Gamma_{0}$ & $\Gamma_{0}$ & $\Gamma_{0}$ & $\begin{array}{l}\Gamma_{0} \\
\Gamma_{1}\end{array}$ & $\begin{array}{l}B, C, D D \\
C, B D\end{array}$ \\
& & & $\Gamma_{2}$ & $B, C, D$ \\
\hline$\Gamma_{2}$ & $\Gamma_{0}$ & $\Gamma_{0}$ & $\Gamma_{0}$ & $C, D$ \\
& & & $\Gamma_{1}$ & $B, C$ \\
\hline$\Gamma_{2}$ & $\Gamma_{2}$ & $\Gamma_{0}$ & $\Gamma_{0}$ & $D, B C$ \\
& & & $\Gamma_{1}$ & $C, D$ \\
\hline$\Gamma_{1}$ & $\Gamma_{0}$ & $\Gamma_{1}$ & $\Gamma_{0}$ & $C$ \\
\hline$\Gamma_{3}$ & $\Gamma_{0}$ & $\Gamma_{1}$ & $\Gamma_{0}$ & $C, B D$ \\
\hline$\Gamma_{3}$ & $\Gamma_{2}$ & $\Gamma_{1}$ & $\Gamma_{0}$ & $B D$ \\
\hline$\Gamma_{1}$ & $\Gamma_{2}$ & $\Gamma_{1}$ & $\Gamma_{0}$ & $B C D$ \\
\hline$\Gamma_{0}$ & $\Gamma_{2}$ & $\Gamma_{0}$ & $\Gamma_{0}$ & $B, D$ \\
& & & $\Gamma_{1}$ & $B C, C D$
\end{tabular}

Table 2.30

\begin{tabular}{llll|ll}
$P_{A}$ & $P_{B}$ & $P_{C}$ & $P_{F}$ & & \\
\hline$\Gamma_{0}$ & $\Gamma_{0}$ & $\Gamma_{0}$ & $\Gamma_{0}$ & $\Gamma_{0}$ & $A, B, C, F$ \\
& & & & $\Gamma_{1}$ & $A, B, C, F$ \\
\hline$\Gamma_{0}$ & $\Gamma_{0}$ & $\Gamma_{0}$ & $\Gamma_{1}$ & $\Gamma_{0}$ & $A, B, C$
\end{tabular}


Table 2.31

\begin{tabular}{lcccc|cl}
$P_{A}$ & $P_{B}$ & $P_{C}$ & $P_{D}$ & $P_{I}$ & & \\
\hline$\Gamma_{0}$ & $\Gamma_{0}$ & $\Gamma_{0}$ & $\Gamma_{0}$ & $\Gamma_{0}$ & $\begin{array}{l}\Gamma_{0} \\
\Gamma_{1}\end{array}$ & $\begin{array}{l}A, B, C, D, I \\
A, B, C, D, I\end{array}$ \\
\hline$\Gamma_{0}$ & $\Gamma_{0}$ & $\Gamma_{0}$ & $\Gamma_{1}$ & $\Gamma_{0}$ & $\Gamma_{0}$ & $A, B, C, I$ \\
\hline$\Gamma_{0}$ & $\Gamma_{0}$ & $\Gamma_{0}$ & $\Gamma_{0}$ & $\Gamma_{1}$ & $\Gamma_{0}$ & $A, B, C, D$ \\
\hline$\Gamma_{0}$ & $\Gamma_{0}$ & $\Gamma_{0}$ & $\Gamma_{1}$ & $\Gamma_{1}$ & $\Gamma_{0}$ & $A, B, C, D I$
\end{tabular}

Table 2.29

\begin{tabular}{|c|c|c|c|c|}
\hline$P_{B}$ & $P_{C}$ & $P_{D}$ & & \\
\hline$\Gamma_{0}$ & $\Gamma_{0}$ & $\Gamma_{0}$ & $\begin{array}{l}\Gamma_{0} \\
\Gamma_{1} \\
\Gamma_{2} \\
\Gamma_{3}\end{array}$ & $\begin{array}{l}B, C, D \\
C, B D \\
C, B D \\
B, C, D\end{array}$ \\
\hline$\Gamma_{0}$ & $\Gamma_{0}$ & $\Gamma_{3}$ & $\begin{array}{l}\Gamma_{0} \\
\Gamma_{2}\end{array}$ & $\begin{array}{l}B, C \\
C\end{array}$ \\
\hline$\Gamma_{0}$ & $\Gamma_{3}$ & $\Gamma_{0}$ & $\begin{array}{l}\Gamma_{0} \\
\Gamma_{2}\end{array}$ & $\begin{array}{l}B, D \\
B D\end{array}$ \\
\hline$\Gamma_{3}$ & $\Gamma_{0}$ & $\Gamma_{0}$ & $\begin{array}{l}\Gamma_{0} \\
\Gamma_{2}\end{array}$ & $\begin{array}{l}C, D \\
C\end{array}$ \\
\hline$\Gamma_{3}$ & $\Gamma_{3}$ & $\Gamma_{0}$ & $\begin{array}{l}\Gamma_{0} \\
\Gamma_{2}\end{array}$ & $\begin{array}{l}D, B C \\
B C D\end{array}$ \\
\hline$\Gamma_{3}$ & $\Gamma_{0}$ & $\Gamma_{3}$ & $\begin{array}{l}\Gamma_{0} \\
\Gamma_{2} \\
\Gamma_{4}\end{array}$ & $\begin{array}{l}C, B D \\
C, B D \\
C, B D\end{array}$ \\
\hline$\Gamma_{0}$ & $\Gamma_{3}$ & $\Gamma_{3}$ & $\begin{array}{l}\Gamma_{0} \\
\Gamma_{2}\end{array}$ & $\begin{array}{l}B, C D \\
B C D\end{array}$ \\
\hline$\Gamma_{3}$ & $\Gamma_{3}$ & $\Gamma_{3}$ & $\begin{array}{l}\Gamma_{0} \\
\Gamma_{2}\end{array}$ & $\begin{array}{l}B C, C D \\
B D\end{array}$ \\
\hline
\end{tabular}

Table 2.33

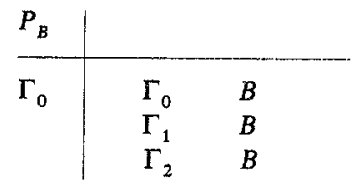

Table 2.34

\begin{tabular}{l|ll}
$P_{B}$ & & \\
\hline$\Gamma_{0}$ & $\Gamma_{0}$ & $B$ \\
& $\Gamma_{1}$ & $B$ \\
& $\Gamma_{2}$ & $B$ \\
& $\Gamma_{3}$ & $B$ \\
\hline$\Gamma_{2}$ & $\Gamma_{0}$ & $E$ \\
& $\Gamma_{1}$ & $E$
\end{tabular}

Table 2.35

\begin{tabular}{l|ll}
$P_{B}$ & & \\
\hline$\Gamma_{0}$ & $\Gamma_{0}$ & $B$ \\
& $\Gamma_{1}$ & $B$ \\
& $\Gamma_{2}$ & $B$ \\
& $\Gamma_{3}$ & $B$ \\
& $\Gamma_{4}$ & $B$ \\
& $\Gamma_{5}$ & $B$ \\
\hline$\Gamma_{3}$ & $\Gamma_{0}$ & $E$ \\
& $\Gamma_{1}$ & $E$ \\
& $\Gamma_{2}$ & $E$
\end{tabular}

Table 2.36

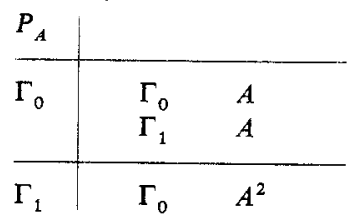


Table 2.39

\begin{tabular}{ll|ll}
$P_{B}$ & $P_{C}$ & & \\
\hline$\Gamma_{0}$ & $\Gamma_{0}$ & $\Gamma_{0}$ & $B, C$ \\
& & $\Gamma_{1}$ & $B, C$ \\
& & $\Gamma_{2}$ & $B, C$ \\
& & $\Gamma_{3}$ & $B, C$ \\
\hline$\Gamma_{2}$ & $\Gamma_{0}$ & $\Gamma_{0}$ & $C$ \\
& & $\Gamma_{1}$ & $C$ \\
\hline$\Gamma_{0}$ & $\Gamma_{2}$ & $\Gamma_{0}$ & $B$ \\
& & $\Gamma_{1}$ & $B$ \\
\hline$\Gamma_{2}$ & $\Gamma_{2}$ & $\Gamma_{0}$ & $B C$ \\
& & $\Gamma_{1}$ & $B C$
\end{tabular}

Table 2.40

\begin{tabular}{ll|ll}
$P_{B}$ & $P_{C}$ & & \\
\hline$\Gamma_{0}$ & $\Gamma_{0}$ & $\Gamma_{0}$ & $B, C$ \\
& & $\Gamma_{1}$ & $B, C$ \\
& & $\Gamma_{2}$ & $B, C$ \\
& & $\Gamma_{3}$ & $B, C$ \\
& & $\Gamma_{3}$ & $B, C$ \\
& & $B, C$ \\
\hline$\Gamma_{3}$ & $\Gamma_{0}$ & $\Gamma_{0}$ & $C$ \\
& & $\Gamma_{1}$ & $C$ \\
& & $\Gamma_{2}$ & $C$ \\
\hline$\Gamma_{0}$ & $\Gamma_{3}$ & $\Gamma_{0}$ & $B$ \\
& & $\Gamma_{1}$ & $B$ \\
& & $\Gamma_{1}$ & $B$ \\
\hline$\Gamma_{3}$ & $\Gamma_{3}$ & $\Gamma_{0}$ & $B C$ \\
& & $\Gamma_{1}$ & $B C$ \\
& & $\Gamma_{2}$ & $B C$
\end{tabular}

Table 2.41

\begin{tabular}{ll|ll}
$P_{A}$ & $P_{B}$ & & \\
\hline$\Gamma_{0}$ & $\Gamma_{0}$ & $\Gamma_{0}$ & $A, B$ \\
& & $\Gamma_{1}$ & $A, B$ \\
\hline$\Gamma_{1}$ & $\Gamma_{0}$ & $\Gamma_{0}$ & $A^{2}, B$ \\
\hline$\Gamma_{0}$ & $\Gamma_{1}$ & $\Gamma_{0}$ & $A$ \\
\hline$\Gamma_{1}$ & $\Gamma_{1}$ & $\Gamma_{0}$ & $A^{2}, A B$
\end{tabular}

Table 2.42

\begin{tabular}{llll|ll}
$P_{A}$ & $P_{B}$ & $P_{C}$ & $P_{C}$ & & \\
\hline$\Gamma_{0}$ & $\Gamma_{0}$ & $\Gamma_{0}$ & $\Gamma_{0}$ & $\Gamma_{0}$ & $A, B, C, D$ \\
& & & & $\Gamma_{1}$ & $A, B, C, D$ \\
\hline$\Gamma_{0}$ & $\Gamma_{0}$ & $\Gamma_{0}$ & $\Gamma_{1}$ & $\Gamma_{0}$ & $A, B, C$
\end{tabular}

Here the unitary representations of the cyclic group $\mathrm{C}_{N}$ (defining relation $A^{N}=E$ ) are denoted by $\Gamma_{k}$ and defined by

$$
\Gamma_{k}\left(A^{m}\right)=\exp (2 \pi \mathrm{i} k m / N) \quad k, m=0, \ldots, N-1 .
$$

As an example of the use of these tables let us consider the group $G=\mathrm{C}_{4} \times \mathrm{C}_{2}$, $G_{0}=\mathrm{C}_{4}$. Here $H=G_{0}, K=\mathrm{C}_{2}$ and $K_{0}=\mathrm{C}_{1} . K$ has two inequivalent factor systems given by $v(B, B)= \pm 1$. For both factor systems there is one irreducible PUA representation of $K: D_{1}(E)=D_{1}(B)=1$ for $v(B, B)=1$ and

$$
D_{2}(E)=\left(\begin{array}{ll}
1 & 0 \\
0 & 1
\end{array}\right), \quad D_{2}(B)=\left(\begin{array}{rr}
0 & 1 \\
-1 & 0
\end{array}\right)
$$

for $v(B, B)=-1$ (see $\S 7$ ). From table 2.17 it follows that $G$ has four inequivalent factor systems, given by $v(B, B)= \pm 1$ and $P_{B}=\Gamma_{0}$ or $P_{B}=\Gamma_{1}$. Consider first the trivial factor system $\left(v(B, B)=1, P_{B}=\Gamma_{0}\right)$. There are three orbits in this case, with representatives $\Gamma_{0}, \Gamma_{1}$ and $\Gamma_{2}$. Further $L\left(\Gamma_{0}\right)=L\left(\Gamma_{2}\right)=G$ and $L\left(\Gamma_{1}\right)=H$. Both $L\left(\Gamma_{0}\right)$ and $L\left(\Gamma_{2}\right)$ have one allowable UA representation $D_{1}$ and $D_{2}$ respectively. They are one dimensional and since $L\left(\Gamma_{0}\right)=L\left(\Gamma_{1}\right)=G$ we do not need equation (3.10). The only allowable representation of $L\left(\Gamma_{1}\right)$ is $\Gamma_{1}$ and inducing this representation to $G$ with equation (3.10) gives a two-dimensional UA representation of $G$. Now consider the factor system given by $v(B, B)=-1$ and $P_{B}=\Gamma_{0}$. Again there are three orbits with representatives $\Gamma_{0}, \Gamma_{1}$ and 
$\Gamma_{2} ; L\left(\Gamma_{0}\right)=L\left(\Gamma_{2}\right)=G$ and $L\left(\Gamma_{1}\right)=H$. For this factor system the allowable PUA representations of $L\left(\Gamma_{0}\right)$ and $L\left(\Gamma_{2}\right)$ are $D_{2}$ and $\Gamma_{2} D_{2}$ respectively; they are two-dimensional PUA representations of $G$. The only allowable PU representation of $L\left(\Gamma_{1}\right)=\Gamma_{1}$, and with equation (3.10) this gives a two-dimensional PUA representation of $G$. So for this factor system $G$ has three two-dimensional PUA representations. Next consider the factor system given by $v(B, B)=1$ and $P_{B}=\Gamma_{1}$. There are two orbits in this case, with representatives $\Gamma_{0}$ and $\Gamma_{2}$. Here $L\left(\Gamma_{0}\right)=L\left(\Gamma_{2}\right)=H$, and the allowable PU representations of $M\left(\Gamma_{0}\right)$ and $M\left(\Gamma_{2}\right)$ are $\Gamma_{0}$ and $\Gamma_{2}$ respectively. From equation (3.10) we obtain two two-dimensional PUA representations of $G$. For the factor system given by $v(B, B)=-1$ and $P_{B}=\Gamma_{1}$ we also obtain two two-dimensional PUA representations of $G$, the only difference from the preceding case being the different factor system in equation (3.10).

Table 3.5

\begin{tabular}{|c|c|c|c|c|c|}
\hline & & $A$ & & $B$ & C \\
\hline Trivial factory system & $\begin{array}{l}D_{1} \\
D_{2} \\
D_{3} \\
D_{4}\end{array}$ & $\begin{array}{rr}1 & \\
1 & \\
1 & \\
1 & 0 \\
0 & -1 \\
0 & 0\end{array}$ & $\left.\begin{array}{r}0 \\
0 \\
-1\end{array}\right)$ & $\left.\begin{array}{rrr}1 & & \\
1 & & \\
1 & & \\
-1 & 0 & 0 \\
0 & 1 & 0 \\
0 & 0 & -1\end{array}\right)$ & $\begin{array}{l}1 \\
\omega \\
\omega^{2} \\
\left(\begin{array}{lll}0 & 0 & 1 \\
1 & 0 & 0 \\
0 & 1 & 0\end{array}\right)\end{array}$ \\
\hline Non-trivial factor system & $D_{5}$ & $\left(\begin{array}{l}i / \sqrt{ } 3 \\
2 i / \sqrt{ } 6\end{array}\right.$ & $\begin{array}{r}2 i / \sqrt{ } 6 \\
-i / \sqrt{ } 3\end{array}$ & $\left(\begin{array}{ll}\mathrm{i} / \sqrt{ } 3 & 2 \mathrm{i} \omega / \sqrt{ } 6 \\
2 \mathrm{i} \omega^{2} / \sqrt{ } 6 & -\mathrm{i} / \sqrt{ } 3\end{array}\right)$ & $\left(\begin{array}{cc}\omega^{2} & 0 \\
& \\
0 & \omega\end{array}\right)$ \\
\hline
\end{tabular}

Table 3,6

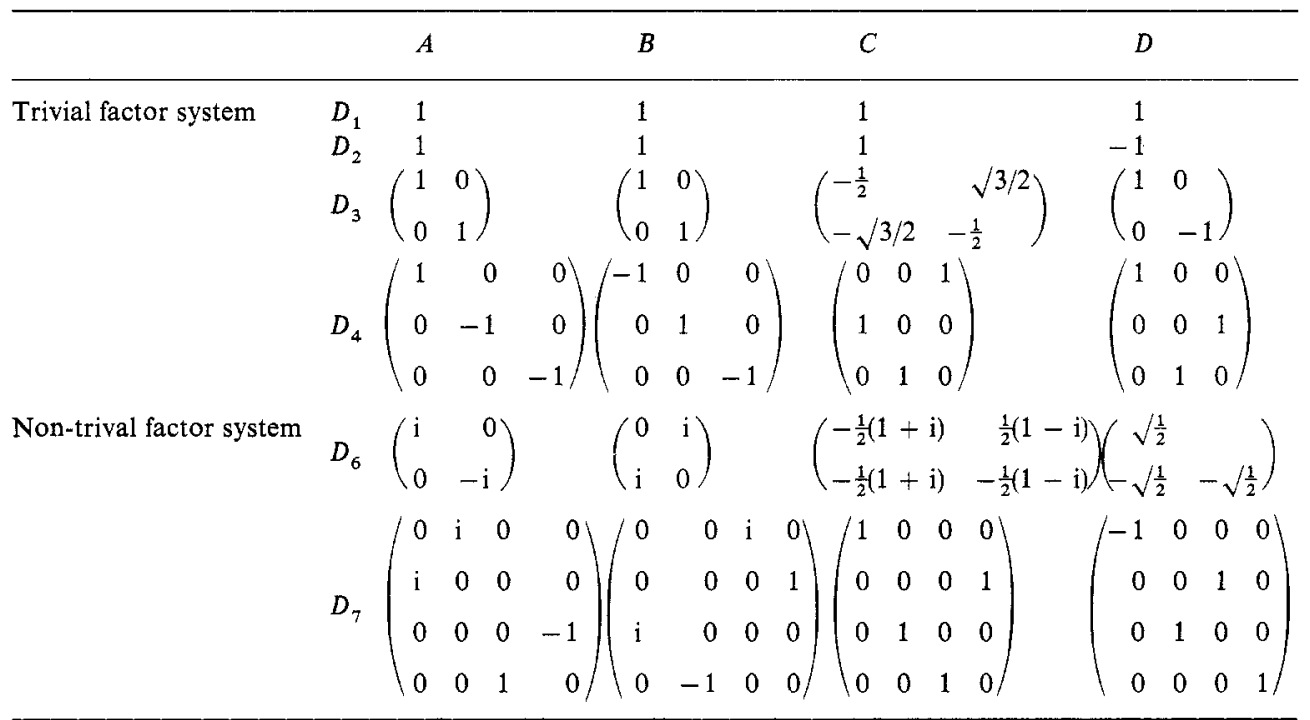

$D_{5}=D_{4} \otimes D_{2} ; D_{8}=D_{6} \otimes D_{2}$. 


\section{The irreducible PU representations of the groups $\mathbf{T}$ and $\mathrm{O}$}

There remain eight Shubnikov point groups to be treated, among which are two ordinary point groups: $\mathrm{T}$ and $\mathrm{O}$. Both $\mathrm{T}$ and $\mathrm{O}$ have two inequivalent factor systems. Their irreducible pu representations are given in tables 3.5 and 3.6 respectively, where matrices for each of their generators are listed. The matrices of the other elements can be calculated by expressing the elements in the generators in alphabetical order: if $g=A^{n} B^{m} C^{p} D^{q}$, then $D_{i}(g)=D_{i}^{n}(A) D_{i}^{m}(B) D_{i}^{p}(C) D_{i}^{q}(D)$. The non-trivial factor system can be calculated from $D(g) D\left(g^{\prime}\right)=\sigma\left(g, g^{\prime}\right) D\left(g g^{\prime}\right)$ for any of the PU representations $D$ with the non-trivial factor system.

\section{Generalised induction and decomposition of factor systems for Shubnikov point groups of type B}

For the Shubnikov point groups of type B we will use the fact that the factor systems and the irreducible PU representations of their non-magnetic subgroups are known. Let $G$ be a finite magnetic group and $G_{0}$ its non-magnetic subgroup of index 2:

$$
G=G_{0}+a G_{0}
$$

where $a$ is a fixed element of $G \backslash G_{0}$. Elements of $G$ are denoted by $h \alpha$, where $h \in G_{0}$ and $\alpha=e$ or $\alpha=a$ ( $e$ is the identity of $G$ ). Each factor system of $G$ is equivalent with a factor system of the form

$$
\sigma\left(h \alpha, h^{\prime} \alpha^{\prime}\right)=\gamma\left(h, \alpha h^{\prime} \alpha^{-1}\right) \gamma\left(h \alpha h^{\prime} \alpha^{-1}, m\left(\alpha, \alpha^{\prime}\right)\right) v\left(\alpha, \alpha^{\prime}\right) P_{\alpha}\left(h^{\prime}\right)
$$

where $\gamma$ is a factor system of $H ; m$ is given by $m(e, e)=m(a, e)=m(e, a)=e$ and $m(a, a)=\dot{a}^{2} ;$ and further

$$
\begin{aligned}
& v(e, e)=v(e, a)=v(a, e)=P_{a}(e)=1 \\
& P_{e}(h)=1 \quad \forall h \in G_{0} \\
& P_{a}\left(h h^{\prime}\right) P_{a}^{*}(h) P_{a}^{*}\left(h^{\prime}\right)=\gamma\left(a h a^{-1}, a h^{\prime} a^{-1}\right) \gamma\left(h, h^{\prime}\right) \quad \forall h, h^{\prime} \in G_{0} \\
& P_{a}^{*}\left(a h a^{-1}\right) P_{a}(h)=\gamma\left(a^{2} h a^{-2}, a^{2}\right) \gamma^{*}\left(a^{2}, h\right) \quad \forall h \in G_{0} \\
& v^{2}(a, a)=P_{a}\left(a^{2}\right) .
\end{aligned}
$$

(This follows from theorem 1 of van den Broek (1976).)

Two factor systems $\sigma$ and $\sigma^{\prime}$ of $G$ which both satisfy equation $(6.2)(\sigma=(\gamma, v, P)$ and $\left.\sigma^{\prime}=\left(\gamma^{\prime}, v^{\prime}, P^{\prime}\right)\right)$ are equivalent if and only if there exists a mapping $d: G_{0} \rightarrow U(1)$ with the properties

$$
\begin{array}{ll}
\gamma^{\prime}\left(h, h^{\prime}\right)=d^{*}(h) d^{*}\left(h^{\prime}\right) d\left(h h^{\prime}\right) \gamma\left(h, h^{\prime}\right) & \forall h, h^{\prime} \in G_{0} \\
P_{a}^{\prime}(h)=d\left(a h a^{-1}\right) d(h) P_{a}(h) & \forall h \in G_{0}
\end{array}
$$

and

$$
v^{\prime}(a, a)=d\left(a^{2}\right) v(a, a) .
$$

(This follows from theorem 3 of van den Broek (1976).) 
The procedure of generalised induction, starting from $G_{0}$, is equal to Wigner's method for the constructuction of the irreducible PUA representations of $G$ from the irreducible PU representations of $G_{0}$ (Murthy 1966, Shaw and Lever 1974).

Suppose $\sigma$ is a factor system of $G$ which satisfies equation (5.2). For each irreducible PU representation $\Gamma$ of $G_{0}$ with factor system $\gamma$ the irreducible PU representation $\tilde{\Gamma}$ of $G_{0}$ is defined by

$$
\tilde{\Gamma}(h)=\Gamma^{*}\left(a^{-1} h a\right) P_{a}^{*}\left(a^{-1} h a\right) .
$$

There are three different cases:

Case I: $\Gamma$ is equivalent with $\tilde{\Gamma} ; \Gamma(h)=U \tilde{\Gamma}(h) U^{-1}$ and $U U^{*}=v(a, a) \Gamma\left(a^{2}\right)$ for some unitary matrix $U$,

Case II: $\Gamma$ is equivalent with $\tilde{\Gamma} ; \Gamma(h)=U \tilde{\Gamma}(h) U^{-1}$ and $U U^{*}=-v(a, a) \Gamma\left(a^{2}\right)$ for some unitary matrix $U$,

Case III. $\Gamma$ and $\tilde{\Gamma}$ are inequivalent.

For each $\Gamma$ of case I or case II and each pair $(\Gamma, \tilde{\Gamma})$ of case III there is one irreducible PUA representation $D$ of $G$ with factor system $\sigma$ :

Case I: $\quad D(h)=\Gamma(h)$

Case II: $D(h)=\left(\begin{array}{ll}\Gamma(h) & 0 \\ 0 & \Gamma(h)\end{array}\right)$

Case III: $D(h)=\left(\begin{array}{ll}\Gamma(h) & 0 \\ 0 & \tilde{\Gamma}(h)\end{array}\right)$

$$
\forall h \in G_{0} ; \quad D(a)=U,
$$$$
\forall h \in G_{0} ; \quad D(a)=\left(\begin{array}{cc}
0 & U \\
-U & 0
\end{array}\right),
$$

$$
\forall h \in G_{0} ; \quad D(a)=\left(\begin{array}{ll}
0 & v(a, a) \Gamma\left(a^{2}\right) \\
1 & 0
\end{array}\right)
$$

So the following information is needed in order to be able to write down the matrices of all the irreducible PUA representations of $G$ :

(i) From the complete set of inequivalent factor systems of $G$ the subset of factor systems with the property that the equations (6.5) and (6.6) have solutions.

(ii) For each factor system of this subset a particular solution $P_{a}^{\prime}$ of the equations (6.5) and (6.6).

(iii) For each factor system of this subset a set of pairs $(D, v(a, a))$, where $D$ is a onedimensional unitary representation of $G_{0}$, such that with $P_{a}=P_{a}^{\gamma} D$ equation (6.2) gives a complete set of inequivalent factor systems of $G$.

(iv) For each of these factor systems of $G$ and for each irreducible PU representation $\Gamma$ of $G_{0}$ with this factor system the case $\Gamma$ belongs to; moreover, if $\Gamma$ belongs to case I or II and is at least two-dimensional a matrix $U$ satisfying $\Gamma(h)=U \tilde{\Gamma}(h) \mathrm{U}^{-1}$ for each $h \in G_{0}$.

For the Shubnikov point groups of type $B$ this information will be given in the next section.

\section{Tables for the irreducible PUA representations of the Shubnikov point griups of type B}

For each of the six Shubnikov point groups of type B each factor system of the nonmagnetic subgroup has the property that the equations (6.5) and (6.6) have solutions. The particular solution $P_{a}^{\gamma}$ of the equations (6.5) and (6.6) can be taken equal to 1 if $\gamma$ is the trivial factor system and also for the non-trivial factor systems for the groups $T \times C_{2}(T)$ and $\mathrm{O} \times \mathrm{C}_{2}(\mathrm{O})$ (with the choice $a=D$ and $a=F$ respectively). For the group $\mathrm{D}_{4}\left(\mathrm{D}_{2}\right)$ and the non-trivial factor system of $\mathrm{D}_{2}$ given by table 2.1 (with $A$ replaced by $A^{2}$ ) we take $a=A B, P_{A B}^{\gamma}(E)=P_{A B}^{\gamma}\left(A^{2}\right)=1$ and $P_{A B}^{\gamma}(B)=P_{A B}^{\gamma}\left(A^{2} B\right)=\mathrm{i}$. For the group $\mathrm{O}(\mathrm{T})$ 
Table 4.14

\begin{tabular}{l|l|l}
$v(A, A)=1$ & $\Gamma_{0}$ & $\mathrm{I}$ \\
\hline$v(A, A)=-1$ & $\Gamma_{0}$ & II
\end{tabular}

\section{Table 4.32}

\begin{tabular}{l|l|l}
$P_{A}=\Gamma_{0}$ & $\Gamma_{0}$ & I \\
$v(A, A)=1$ & $\Gamma_{1}$ & II \\
\hline \begin{tabular}{l|l}
$P_{A}=\Gamma_{1}$ \\
$v(A, A)=\mathrm{i}$
\end{tabular} & $\Gamma_{0}, \Gamma_{1}$ & III
\end{tabular}

\begin{tabular}{l|l|l|l} 
Table 4.23 & & \\
$\gamma$ trivial & $D_{1}$ & I & \\
$P_{D}=D_{1}$ & $D_{2}, D_{3}$ & III & \\
$v(D, D)=1$ & $D_{4}$ & I & $e_{3}$ \\
\hline$\gamma$ trivial & $D_{1}$ & II & \\
$P_{D}=D_{1}$ & $D_{2}, D_{3}$ & III & \\
$v(D, D)=-1$ & $D_{4}$ & II & $e_{3}$ \\
\hline$\gamma$ non-trivial & $D_{5}$ & II & $p$ \\
$P_{D}=D_{1}$ & $D_{6}, D_{7}$ & III & \\
$v(D, D)=1$ & & & \\
\hline$\gamma$ non-trivial & $D_{5}$ & I & $p$ \\
$P_{D}=D_{1}$ & $D_{6}, D_{7}$ & III & \\
$v(D, D)=-1$ & & &
\end{tabular}

Table 4,37.1. Irreducible projective representations of $\mathrm{D}_{2}$ $\gamma$ trivial

\begin{tabular}{l|rrrr} 
& $E$ & $A^{2}$ & $B$ & $A^{2} B$ \\
\hline$D_{1}$ & 1 & 1 & 1 & 1 \\
$D_{2}$ & 1 & 1 & -1 & -1 \\
$D_{3}$ & 1 & -1 & 1 & -1 \\
$D_{4}$ & 1 & -1 & -1 & 1
\end{tabular}

$\gamma$ non-trivial

\begin{tabular}{|c|c|c|c|c|}
\hline & $E$ & $A^{2}$ & $B$ & $A^{2} B$ \\
\hline$D_{5}$ & & $(1$ & 0 & $\left(\begin{array}{ll}0 & 1\end{array}\right.$ \\
\hline
\end{tabular}

Table 4.24

$\gamma$ trivial

$P_{F}=D_{1}$

$v(F, F)=1$

\begin{tabular}{l|l|l|l} 
& $D_{3}$ & I & $e_{2}$ \\
& $D_{4}$ & I & $e_{3}$ \\
& $D_{5}$ & I & $e_{3}$ \\
\hline$\gamma$ trivial & $D_{1}$ & II &
\end{tabular}

$P_{F}=D_{1}$

$v(F, F)=-1$

\begin{tabular}{l|l|l|l} 
& $D_{3}$ & II & $e_{2}$ \\
& $D_{5}$ & II & $e_{3}$ \\
\hline$\gamma$ trivial & $D_{1}, D_{2}$ & III & \\
$P_{F}=D_{2}$ & $D_{3}$ & II & $p$ \\
$\nu(F, F)=1$ & $D_{4}, D_{5}$ & III & \\
\hline$\gamma$ trivial & $D_{1}, D_{2}$ & III & \\
$P_{F}=D_{2}$ & $D_{3}$ & I & $p$ \\
$\nu(F, F)=-1$ & $D_{4}, D_{5}$ & III & \\
\hline$\gamma$ non-trivial & $D_{6}, D_{8}$ & III & \\
$P_{F}=D_{1}$ & $D_{7}$ & I & $q$ \\
$v(F, F)=1$ & & & \\
\hline$\gamma$ non-trivial & $D_{6}, D_{8}$ & III & \\
$P_{F}=D_{1}$ & $D_{7}$ & II & $q$ \\
$\nu(F, F)=-1$ & & & \\
\hline$\gamma$ non-trivial & $D_{6}$ & II & $p$ \\
$P_{F}=D_{2}$ & $D_{7}$ & II & $r$ \\
$\nu(F, F)=1$ & $D_{8}$ & II & $p$ \\
\hline$\gamma$ non-trivial & $D_{6}$ & I & $p$ \\
$P_{F}=D_{2}$ & $D_{7}$ & I & $r$ \\
$\nu(F, F)=-1$ & $D_{8}$ & I & $p$
\end{tabular}


Table 4.37.2

$\gamma$ trivial

$P_{A B}=D_{1}$

$v(A B, A B)=1$

$\gamma$ trivial

$P_{A B}=D_{1}$

$v(A B, A B)=-1$

$\gamma$ non-trivial

$P_{A B}=P_{A B}^{\gamma} D_{1}$

$\nu(A B, A B)=1$

$\gamma$ non-trivial

$P_{A B}=P_{A B}^{y} D_{1}$

$v(A B, A B)=-1$

\begin{tabular}{|l|l|l}
$D_{1}$ & I & \\
$D_{2}$ & I & \\
$D_{3}, D_{4}$ & III & \\
\hline$D_{1}$ & II & \\
$D_{2}$ & II & \\
$D_{3}, D_{4}$ & III & \\
\hline$D_{5}$ & I & $s$ \\
& & \\
\hline$D_{5}$ & II & $s$ \\
& &
\end{tabular}

Table 4.38

$\gamma$ trivial

$P_{D}=D_{1}$

$v(D, D)=1$

\begin{tabular}{l|c|c|c}
$\gamma(D, D)=1$ & $D_{3}$ & I & \\
& $D_{4}$ & I & $u$ \\
\hline$\gamma$ trivial & $D_{1}$ & II & \\
$P_{D}=D_{1}$ & $D_{2}$ & II & \\
$v(D, D)=-1$ & $D_{3}$ & II & \\
& $D_{4}$ & II & $u$ \\
\hline$\gamma$ non-trivial & $D_{5}$ & I & $t$ \\
$P_{D}=P_{D}^{\gamma} D_{1}$ & $D_{6}$ & I & $t$ \\
$\gamma(D, D)=1$ & $D_{7}$ & I & $t$ \\
\hline$\gamma$ non-trivial & $D_{5}$ & II & $t$ \\
$P_{D}=P_{D}^{\gamma} D_{1}$ & $D_{6}$ & II & $t$ \\
$v(D, D)=-1$ & $D_{7}$ & II & $t$
\end{tabular}

and the non-trivial factor system of T given by the table 3.5 we take $a=D, P_{D}^{\gamma}(E)=$ $P_{D}^{\gamma}(C)=P_{D}^{\gamma}\left(C^{2}\right)=1$ and $P_{D}^{\gamma}(h)=-1$ for $h \in T \backslash\left\{E, C, C^{2}\right\}$.

Table 4 contains for each of the Shubnikov point groups of type $B$ the following information: the inequivalent factor systems are given in the first column, specified by the factor system $\gamma$ of $G_{0}$, the mapping $P_{a}: G_{0} \rightarrow U(1)$ and $\nu(a, a)$; for each factor system of $G$ the irreducible PU representations of $G_{0}$ are listed in the second column, where the pairs $(\Gamma, \tilde{\Gamma})$ of case III have been put together; in the third column the case is given to which the irreducible PU representation of $G_{0}$ belongs and, if $\Gamma$ belongs to case I or case II and is at least two-dimensional, in the fourth column a matrix $U$ is given which satisfies $\Gamma(h)=U \tilde{\Gamma}(h) U^{-1}$ for all $h \in G_{0}$. The matrices occurring in the last columns of these tables are

$$
\begin{aligned}
& e_{2}=\left(\begin{array}{ll}
1 & 0 \\
0 & 1
\end{array}\right) ; \quad e_{3}=\left(\begin{array}{lll}
1 & 0 & 0 \\
0 & 1 & 0 \\
0 & 0 & 1
\end{array}\right) ; \quad p=\left(\begin{array}{cc}
0 & 1 \\
-1 & 0
\end{array}\right) ; \quad s=\left(\begin{array}{cc}
1 & 0 \\
0 & -\mathrm{i}
\end{array}\right) ; \quad t=\left(\begin{array}{cc}
1 & 0 \\
0 & \omega^{2}
\end{array}\right) ; \\
& u=\left(\begin{array}{lll}
0 & 1 & 0 \\
1 & 0 & 0 \\
0 & 0 & 1
\end{array}\right) ; \quad q=\left(\begin{array}{rrrr}
1 & 0 & 0 & 0 \\
0 & -1 & 0 & 0 \\
0 & 0 & -1 & 0 \\
0 & 0 & 0 & -1
\end{array}\right) \text { and } r=\frac{1}{\sqrt{3}}\left(\begin{array}{rccc}
0 & 1 & 1 & 1 \\
-1 & 0 & -\mathrm{i} & \mathrm{i} \\
-1 & \mathrm{i} & 0 & -\mathrm{i} \\
-1 & -\mathrm{i} & \mathrm{i} & 0
\end{array}\right)
\end{aligned}
$$

\section{Acknowledgment}

I thank Dr T Janssen for reading the manuscript.

\section{Appendix}

It will often occur that if one wants to know the irreducible PUA representations of a Shubnikov point group $G$ with a factor system $\omega$ this factor system is not equal to one of the factor systems used in the preceding tables. Suppose $\omega$ is equivalent with the factor 
system $\sigma$ which does occur in the tabulation. Then

$$
\sigma\left(g, g^{\prime}\right)=\frac{c(g) c^{g}\left(g^{\prime}\right)}{c\left(g g^{\prime}\right)} \omega\left(g, g^{\prime}\right) \quad \forall g \in G
$$

for some mapping $c: G \rightarrow U(1)$. One has to determine $\sigma$ and the mapping $c$. Then the irreducible PUA representations $D_{\omega}$ of $G$ with factor system $\omega$ are given by

$$
D_{\omega}(g)=c^{*}(g) D_{\sigma}(g)
$$

where $D_{\sigma}$ are the irreducible PUA representations of $G$ with factor system $\sigma$ which may be obtained from the previous sections. First suppose that $G$ is a Shubnikov point group of type A. Let us define the factor system $\omega^{\prime}$ of $G$, which is equivalent with $\omega$, by

$$
\omega^{\prime}\left(h k, h^{\prime} k^{\prime}\right)=\frac{p(h k) p^{k}\left(h^{\prime} k^{\prime}\right)}{p\left(h k h^{\prime} k^{\prime}\right)} \omega\left(h k, h^{\prime} k^{\prime}\right)
$$

where the mapping $p: G \rightarrow U(1)$ is given by

$$
p(h k)=\omega(h, k) \text {. }
$$

Since $H$ is a finite cyclic group the restriction of $\omega^{\prime}$ to $H \times H$ is a trivial factor system of $H$ and we can write

$$
\omega^{\prime}\left(h, h^{\prime}\right)=\frac{r(h) r\left(h^{\prime}\right)}{r\left(h h^{\prime}\right)} .
$$

If the order of $H$ is $N$ and the elements of $H$ are denoted by $E, A, A^{2}, \ldots A^{N-1}$ the mapping $r: H \rightarrow U(1)$ is given by $r(E)=1$,

$$
r(A)^{N}=\prod_{n=1}^{N-1} \omega^{\prime}\left(A^{n}, A\right)
$$

and

$$
r\left(A^{m}\right)=r(A)^{m} \prod_{n=0}^{m-1} \omega^{*}\left(A^{n}, A\right) \quad m=2, \ldots N-1
$$

where one of the $N$ possible solutions for $r(A)$ of equation (A6) may be chosen arbitrarily.

Now let us extend the mapping $r$ to $G$ with the definition $r(h k)=r(h)$ and let us define the factor system $\omega^{\prime \prime}$ of $G$, which is equivalent to $\omega^{\prime}$, by

$$
\omega^{\prime \prime}\left(h k, h^{\prime} k^{\prime}\right)=\frac{r^{*}(h k) r^{* k}\left(h^{\prime} k^{\prime}\right)}{r^{*}\left(h k h^{\prime} k^{\prime}\right)} \omega^{\prime}\left(h k, h^{\prime} k^{\prime}\right) .
$$

This factor system has the properties

$$
\omega^{\prime \prime}\left(h, h^{\prime}\right)=1 \quad \forall h, h^{\prime} \in H
$$

and

$$
\omega^{\prime \prime}(h, k)=1 \quad \forall h \in H, \forall k \in K .
$$

From this it follows (van den Broek 1976) that

$$
\omega^{\prime \prime}\left(h k, h^{\prime} k^{\prime}\right)=v\left(k, k^{\prime}\right) P_{k}^{\prime}\left(h^{\prime}\right)
$$

where $v$ is a factor system of $K$ and $\left\{P_{k}^{\prime}\right\}$ is a set of one-dimensional representations of $H$ which satisfies equation (3.5). So $w^{\prime \prime}$ has the form of equation (3.2), but the set $\left\{P_{k}^{\prime}\right\}$ is not 
necessarily one of the tabulated sets $\left\{P_{k}\right\}$. However, there always exists a one-dimensional representation $q$ of $H$ such that

$$
P_{k}(h)=q^{k}(h) q^{*}\left(k h k^{-1}\right) P_{k}^{\prime}(h)
$$

where the set $\left\{P_{k}\right\}$ does occur in the tabulation. If we extend $q$ to $G$ by $q(h k)=q(h)$ and define the factor system $\sigma$ by

$$
\sigma\left(h k, h^{\prime} k^{\prime}\right)=\frac{q(h k) q^{k}\left(h^{\prime} k^{\prime}\right)}{q\left(h k h^{\prime} k^{\prime}\right)} \omega^{\prime \prime}\left(h k, h^{\prime} k^{\prime}\right)
$$

then we have

$$
\sigma\left(h k, h^{\prime} k^{\prime}\right)=v\left(k, k^{\prime}\right) P_{k}\left(h^{\prime}\right) .
$$

Moreover $\sigma$ is equivalent with $\omega$ and the mapping $c: G \rightarrow U(1)$ of equation (A1) is given by

$$
c(h k)=q(h) r^{*}(h) \omega(h, k)
$$

For the Shubnikov point groups of type B we may proceed in an analogous way. We define the factor system $\omega^{\prime}$ of $G$ by

$$
\omega^{\prime}\left(h \alpha, h^{\prime} \alpha^{\prime}\right)=\frac{p(h \alpha) p^{\alpha}\left(h^{\prime} \alpha^{\prime}\right)}{p\left(h \alpha h^{\prime} \alpha^{\prime}\right)} \omega\left(h \alpha, h^{\prime} \alpha^{\prime}\right)
$$

where the mapping $p: G \rightarrow U(1)$ now is given by

$$
p(h \alpha)=\omega(h, \alpha) .
$$

The restriction of $\omega^{\prime}$ to $G_{0} \times G_{0}$ can be related to a factor system $\gamma$ of $G_{0}$ occurring in the tables.

$$
\omega^{\prime}\left(h, h^{\prime}\right)=\frac{r(h) r\left(h^{\prime}\right)}{r\left(h h^{\prime}\right)} \gamma\left(h, h^{\prime}\right) .
$$

If we extend the mapping $r$ to $G$ with the definition $r(h \alpha)=r(h)$ and define the factor system $\omega^{\prime \prime}$ of $G$ by

$$
\omega^{\prime \prime}\left(h \alpha, h^{\prime} \alpha^{\prime}\right)=\frac{r^{*}(h \alpha) r^{*^{*}}\left(h^{\prime} \alpha^{\prime}\right)}{r^{*}\left(h \alpha h^{\prime} \alpha^{\prime}\right)} \omega^{\prime}\left(h \alpha, h^{\prime} \alpha^{\prime}\right)
$$

then

$$
\omega^{\prime \prime}\left(h, h^{\prime}\right)=\gamma\left(h, h^{\prime}\right) \quad \forall h, h^{\prime} \in G_{0}
$$

and

$$
\omega^{\prime \prime}(h, a)=1 \quad \forall h \in G_{0} .
$$

Therefore we have (van den Broek 1976)

$$
\omega^{\prime \prime}\left(h \alpha, h^{\prime} \alpha^{\prime}\right)=\gamma\left(h, \alpha h^{\prime} \alpha^{-1}\right) \gamma\left(h \alpha h^{\prime} \alpha^{-1}, m\left(\alpha, \alpha^{\prime}\right)\right) v^{\prime}\left(\alpha, \alpha^{\prime}\right) P_{\alpha}^{\prime}\left(h^{\prime}\right)
$$

where $v^{\prime}$ and $P^{\prime}$ have the properties mentioned in $\S 6$. If $v^{\prime}$ and $P^{\prime}$ do not occur in the tabulation then there exists a one-dimensional representation $q$ of $H$ such that

$$
P_{a}^{\prime}(h)=q\left(a h a^{-1}\right) q(h) P_{a}(h)
$$

and

$$
v^{\prime}(a, a)=q\left(a^{2}\right) v(a, a)
$$

and $P$ and $v$ do occur in the tabulation. Extending $q$ to $G$ by $q(h \alpha)=q(h)$ and defining 
the factor system $\sigma$ by

$$
\sigma\left(h \alpha, h^{\prime} \alpha^{\prime}\right)=\frac{q(h \alpha) q^{\alpha}\left(h^{\prime} \alpha^{\prime}\right)}{q\left(h \alpha h^{\prime} \alpha^{\prime}\right)} \omega^{\prime \prime}\left(h \alpha, h^{\prime} \alpha^{\prime}\right)
$$

we find that $\sigma$ satisfies equation (5.2) and is equivalent with $\omega$; the mapping $c: G \rightarrow U(1)$ of equation (A1) is given by

$$
c(h \alpha)=p(h \alpha) r^{*}(h \alpha) q(h \alpha)
$$

\section{References}

Bradley C J and Cracknell A P 1972 The Mathematical Theory of Symmetry in Solids (Oxford: Clarendon) van den Broek P M 1976,J. Phys. A: Math. Gen. $9855-62$

- 1977 J. Phys. A: Math. Gen. $101653-63$

Hurley A C 1966 Phil. Trans. R. Soc. A $2601-36$

Murthy M V 1966 J. Math. Phys. $7853-7$

Shaw R and Lever J 1976 Commun. Math. Phys. 38 257-77

Tam W G 1969 Physica 42 557-64 